\title{
Degradação de Fármacos e Impacto Ambiental
}

\author{
Euzani S. Duarte, Gislene C. S. de Aquino \& Rogério G. Lima
}

O consumo de medicamentos tem aumentado nos últimos tempos e, com isso, as indústrias têm investido cada vez mais em tecnologias, resultando no acréscimo da geração de resíduos farmacológicos, devido à sua degradação, ocasionando várias consequências ao meio ambiente, devido ao descarte incorreto pela população e pelas indústrias. Uma das preocupações das indústrias é com a questão da estabilidade farmacológica, mas os resíduos produzidos por eles são pouco estudados e estes quando lançados no ambiente contaminam os corpos hídricos, o solo e causam alterações aos seres vivos. Este trabalho busca abordar os aspectos socioeconômicos e ambientais relacionados ao descarte de medicamentos, estabelecendo quais os principais problemas ocasionados pelo descarte incorreto, bem como a legislação acerca da destinação dos resíduos farmacêuticos, para isso serão realizados uma revisão bibliográfica no Science American. Muitos medicamentos são descartados de maneira errônea pela população e indústrias, causando, por exemplo, uma disfunção hormonal em peixes, uma resistência em bactérias. A legislação a respeito do tema é extensa, mas há falhas na sua execução e fiscalização, bem como na criação de uma legislação para a coleta de fármacos vencidos ou inutilizados pela população.

Palavras- Chave: Resíduos farmacológico, degradação, meio ambiente, legislação.

The medicine consumption has been largely increased over last years. It has taken the industry to invest in new technologies which bring in a increasement in the pharmacological waste production due to its degradation which brings a lot of consequences to the environment occasioned by the wrong discard both from population and from industries. The highest concern of the industries is related to the pharmological estability however the questiono fwaste which is produced is not larged argueed. When these wastes are wrongly discarded in the environment they can contamine the hydric resourses, the land and drive many changes to the living beings. This work aims to approach both the socio-economic and environmental aspects that are related to the drug discard attempting to establish what are the main problems occasioned by the wrong discard it is also aimed to discuss the current legislation about this issue. Seeking it, a bibliographical review has been done on the Science American. Lots of medicine are wrongly discarded by the population and industry causing, for instance, the fish hormonal disfunction and bacterias resistance. There is a long legislation about this issue however should be highlighted that there are some fails both in its application and at its supervision and in which is related to the a new legislation that rules the discard of expired or non-used drugs from the population

Key-words: pharmacological waste, degradation, environment, legislation. 


\section{Introdução}

O aumento populacional está associado ao aumento do nível de vida que tem contribuído com o elevado consumo de medicamentos e consequentemente com o aumento na diversidade de produtos com componentes e materiais de difícil degradação e maior toxicidade. O Brasil está entre os maiores consumidores de medicamentos do mundo. Contudo, essa produção de medicamentos, muitas vezes, provoca um grande acúmulo de resíduos sólidos no ambiente. A baixa volatilidade desses compostos indica que sua distribuição no meio ambiente acontecerá principalmente por meio de transporte aquoso, mas também através da cadeia alimentar e dispersão (Tambosi, 2008).

Esses resíduos apresentam várias rotas de entrada no meio ambiente, por exemplo, através da eliminação por excreção após a ingestão, injeção ou infusão de medicamentos, a quantidade excretada depende do tipo de medicamento e do indivíduo; da remoção de medicação tópica durante o banho; e da disposição de medicamentos vencidos ou não mais desejados no esgoto ou no lixo comum (Glassmmeyer ST, et al. 2009), chegam às estações de tratamento de esgoto na sua forma original, ativa, sem sofrer alterações do metabolismo no corpo humano. Por mais eficaz que seja a Estação de Tratamento de Esgoto (ETE), pois a completa eliminação dos resíduos irá depender do medicamento que o deu origem e também da tecnologia do tratamento de esgoto, assim colaboram de forma mais eficaz para a contaminação ambiental, agindo em organismos vivos. (Américo et al., 2012). Outro fator de contaminação é a indústria farmacêutica aparece como uma das maiores geradoras de efluentes líquidos, tendo em vista o largo consumo de água para limpeza e manutenção de seus equipamentos. (Napoleão et al. 2011) e o descarte de produtos químicos acabados e inacabados, gerando a necessidade de desenvolvimento de novos processos de tratamento que garantam uma boa eficiência na remoção desses poluentes emergentes.

O descarte inadequado de produtos químicos tem capacidade de colocar em risco e comprometer os recursos naturais e a qualidade de vida das atuais e futuras gerações (Brasil, 2006).

As características químicas dos medicamentos apresentam um risco potencial à saúde pública e ao meio ambiente. Seus resíduos possuem componentes resistentes, de difícil decomposição, que podem contaminar o solo e a água, os quais, muitas vezes, são utilizados para abastecimento, muitos desses contaminantes não são removidos pelos sistemas de tratamento de água convencionais e podem, assim, causar danos irreversíveis à saúde da população.

Atualmente, a população tem adquirido uma maior conscientização em relação aos problemas ambientais e seus efeitos na qualidade de vida, quando verificam algum erro, denunciam para as autoridades para que estas tomem as cabíveis providências, mas, esta é ainda uma pequena parcela da população. Antibióticos, hormônios, antilipêmicos, antiinflamatórios, analgésicos são os fármacos mais comuns no meio ambiente, sendo detectados em esgoto doméstico, águas superficiais e subterrâneas. A Ciprofloxacina, um dos antibióticos mais utilizados, foi encontrado em efluentes de ETE na Suíça, uma concentração $0,37 \mu \mathrm{g}$ L-1; o diclofenaco, um anti-inflamatório em efluente de ETE na Itália 2,47 $\mu \mathrm{g}$ L-1; o hormônio contraceptivo 17a-Etinilestradiol em efluente de ETE no Brasil 5,0 $\mu \mathrm{g} \mathrm{L-1(Melo,} \mathrm{et} \mathrm{al.} \mathrm{2008).}$

Objetivo: Abordar os aspectos socioeconômicos e ambientais, relacionados ao descarte de medicamentos, estabelecendo quais os principais problemas ocasionados pelo descarte incorreto, bem como a legislação acerca da destinação dos resíduos farmacêuticos.

\section{Material e Método}

Estudo qualitativo a partir de uma revisão bibliográfica no Science American utilizando os seguintes termos: degradação de fármacos; legislação para descarte de fármacos; descarte de fármacos e meio ambiente.

\section{ANÁLISE DOS FÁRMACOS}

Por muitos anos, o homem extraiu de forma extensiva os recursos naturais acarretando o descarte de todo o material inutilizado para a aquisição do produto final, contribuindo com a geração de resíduos. Nas áreas da medicina e farmacêutica, a atuação dos profissionais não seria diferente, todos os anos, o volume de medicamentos descartados é enorme devido a prescrições erradas, abandono de tratamento, automedicação, doações de medicamentos e etc, sendo que "nesses fármacos, 
entre 50 e $90 \%$ de sua dosagem permanece inalterada quando excretada e persiste no meio ambiente" (Balbino, 2011).

Pesquisas apontam que a população mundial atinge cerca de 7 bilhões de habitantes, e de acordo com o Programa da ONU para o Meio Ambiente (PNUMA), gera 1,3 bilhão de toneladas de lixo por ano. Os maiores geradores são: Estados Unidos, China, União Europeia, Japão e Brasil. A redução da produção de lixo é tratada com prioridade pela lei federal no Brasil, porém, a realidade é outra. Segundo a Associação Brasileira de Empresas de Limpeza Pública e Resíduos Especiais (ABRELPE), referente a 2010, a produção de lixo no país cresceu seis vezes mais do que a população. E mais: a quantidade de resíduos com destinação inadequada aumentou quase dois milhões de toneladas, em relação a 2009.

Dentre esses resíduos, os gerados pela saúde, os químicos, são de grande risco para o meio ambiente, devido à sua dificuldade de tratamento e destruição. Esses resíduos são classificados pela ANVISA como "Grupo B", ou seja, resíduos que contêm substâncias prejudiciais à saúde pública ou ao meio ambiente, independentemente de suas características de inflamabilidade, corrosividade, reatividade e toxicidade." (Brasil, 2003). Além disso, esses resíduos químicos são divididos em 8 categorias: "B1- resíduos dos medicamentos ou dos insumos farmacêuticos quando vencidos, contaminados, apreendidos para descarte, parcialmente utilizados, e demais medicamentos impróprios para consumo, que oferecem risco; B2- se diferencia do B1- por não oferecer risco devido a seu princípio ativo e forma farmacêutica; B3- resíduos e insumos farmacêuticos dos medicamentos controlados pela Portaria MS 344/98 e suas atualizações; B4- saneantes e desinfetantes; B5- substâncias para revelação de filmes usados em Raio X; B6- resíduos contendo metais pesados; B7- reagentes para laboratório, isolados ou em conjunto; B8- outros resíduos contaminados com substâncias químicas perigosas."

Esses fármacos devido a sua dificuldade de tratamento e destruição, e sua ação sobre o meio ambiente têm recebido uma atenção especial. Vários países, a saber: Alemanha, Reino Unido, Itália desenvolveram pesquisas com o objetivo de detectar seus resíduos no meio ambiente. Foram identificados 36 fármacos diferentes em diversos rios, dentre eles: antilipidêmicos, hidroclorotiazida, carbamazepina, ibuprofeno, benzafibrato, eritromicina, lincomicinas e claritromicina, anágésicoantipiréticos, anti-inflamatório e anti-hiperintensivo, em algumas Estações de Tratamento de Esgoto também tem sido detectada a presença de fármacos, como: ofloxacino, furosemida, atenalol.

No Brasil, pesquisas referentes à qualidade da água potável na região de Campinas, São Paulo, foram identificadas, na água distribuída para consumo para a população, substâncias como dietilftalato, dibutilftalato, cafeína, bisfenol A, estradiol, etinilestradiol e progesterona, compostos que não deveriam ser encontradas na água consumida por seres humanos (Ghiselli, 2010).

Os fármacos empregados com um propósito terapêutico, após atuarem no organismo e serem metabolizados, podem ser excretados como metabólitos, hidrolisados, ou na forma original. O metabolismo elimina moléculas de droga em excesso, assim como outros compostos tóxicos que não pertencem ao organismo e que sofrem uma série de biotransformações enzimáticas e os converte em compostos mais polares e hidrofilicos (Ikehata et al., 2006). Podem ainda estar conjugados com moléculas polares como glicuronídeos, no entanto, esses conjugados são facilmente clivados, disponibilizando substâncias farmacêuticamente ativas nos esgotos domésticos. Por isso, são de dificeis eliminação pelas ETEs nestas, podem ser degradados, adsorvidos ou ainda reativados, como resultado da atividade microbiana (Silva, 2012).

\section{O metabolismo da droga começa com várias reações bioquímicas incluindo hidroxilação, epoxidação, redução e hidrólise, nas quais grupos funcionais são introduzidos ou transformados (fase I). Logo depois, moléculas endógenas, altamente polares, tais como ácido glucurônico, sulfato, e aminoácidos são unidos a drogas ou metabólitos da transformação fase I, para gerar conjugados (fase II), que são solúveis em água e podem ser prontamente excretados na urina ou bila. Certas drogas, agentes médicos não-terapêuticos e xenobióticos não são metabolizados porque são substâncias pobres para a metabolização de enzimas (como citocromo P450) e podem ser eliminados lentamente do corpo sem biotransformação (Tambosi 2008).}

A grande preocupação das indústrias, no momento de criação de um fármaco, é com a questão da estabilidade farmacológica, no entanto, quando esses são degradados e liberados no meio ambiente, combinam com outros compostos e originam moléculas 
instáveis que são tóxicas para o meio biótico e abiótico. Vários estudos demosntraram que diversos fármacos são persistentes no meio ambiente e não são completamente removidos pelas estações de tratamento de esgotos (Sodré et al. 2010), permanecendo por longos períodos, acarretando sérios riscos socioeconômicos e ambientais, representando um risco para a saúde pública e para o meio ambiente, (Balbino, 2011). A produção industrial normalmente orienta-se pela minimização de custos.

Há vários princípios ativos que possuem características de estabilidade e resistência a vários fatores externos como o $\mathrm{pH}$, temperatura, umidade que são importantes para que os medicamentos possam exercer a sua função no organismo e o mantem ativo também no meio ambiente (Lages,2011).

\section{A presença dos resíduos de fármacos no meio ambiente é resultante das quantidades produzidas e usadas, $d a$ frequência de administração, da taxa de excreção dos fármacos ou metabólitos, da propensão da substância em ser adsorvida em solos e da capacidade de degradação do meio em que o resíduo se encontra. Outro fator a ser considerado é que a facilidade de dispersão destes resíduos, através do transporte aquoso, está associada à natureza polar e à baixa volatilidade destes compostos. Isto dificulta o escape do meio aquático, como presumivelmente ocorre com os compostos orgânicos voláteis (Vieira, 2011).}

Entre os impactos ambientais mais preocupantes associados a resíduos de fármacos está a genotoxicidade (Gil e Mathias, 2005), que pode ser definida como a capacidade que algumas substâncias químicas apresentam em produzir alterações genéticas em organismos a elas expostos.

As propriedades físicoquímicas que os fármacos apresentam como sua característica lipofilica, bioacumulativos e baixa pressão de vapor, contribui para a sua dispersão (Torres et al., 2012). Além disso, apresentam facilidade em atravessar a membrana plasmática das células dos animais, já que essas são lipoproteicas. Portanto, têm sido detectados em diversas matrizes ambientais, por meio de técnicas analíticas, as quais se tornaram mais sensíveis e mais abrangentes. Para determinação de compostos farmacológicos em amostras ambientais os métodos mais utilizados, baseiam-se na extração em fase sólida seguida por cromatografia líquida que é um método de separação, no qual os constituintes da amostra a serem separados são particionados entre duas fases, uma estacionária, geralmente de grande área, e outra um fluído que flui através da primeira. (Torres et al., 2012), cromatografia análises de carbono orgânico total caracteriza pela determinação da taxa de mineralização (transformação dos produtos em $\mathrm{CO}_{2}$ e $\mathrm{H}_{2} \mathrm{O}$ ) de um processo de degradação aplicado em moléculas orgânicas (Vieira, 2011), espectrofotometria de absorção molecular UVvisível e espectrometria de massas.

A deposição de fármacos diretamente no lixo comum é mais prejudicial ao meio ambiente do que a excreção pelo organismo, pois fármacos descartados encontram-se na sua forma ativa. Mesmo as drogas que possuem meia vida curta, são passíveis de causar exposições crônicas, uma vez que sua introdução contínua no meio ambiente leva à bioacumulação (Andreozzi e Rafaelli, 2003). Além disso, mesmo que a concentração de alguns fármacos encontrados no meio ambiente seja baixa, a combinação deles pode ter efeitos pronunciados devido os mecanismos de ação sinérgicas (Reis Filho e Barreto, 2007).

A fluoxetina, por exemplo, é um antidepressivo mais usado comum de ser encontrado nos recursos hídricos é um pó cristalino branco solúvel em água com meia vida de eliminação de 4 a 6 dias, é metabolizada no figado originando a norfluoxetina, meia vida de 4 a 16 dias, entre outros que são excretados na urina (Merck, 2013 apoud Silva 2014). Apresenta baixa biodegrabilidade e alto poder de bioacumulação. Pode ser detectada pela cromatografia líquida de alta eficiência, em que uma mistura de compostos é injetada na coluna e são separadas e em seguida detectados eletronicamente

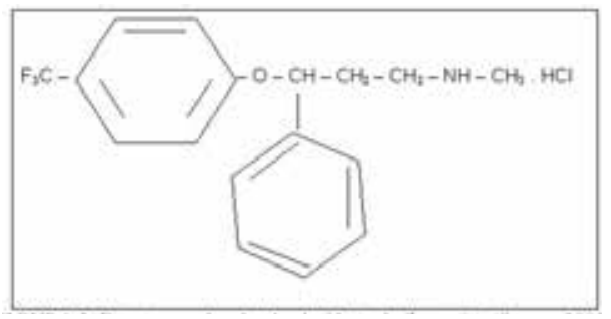

Figura1. Estrutura molecular do cloridrato de fluoxetina (Silva, 2014).

(Silva, 2014).

A trimetoprima é um dos antibióticos mais resistentes em relação aos processos de degradação principalmente fotocatálise, 


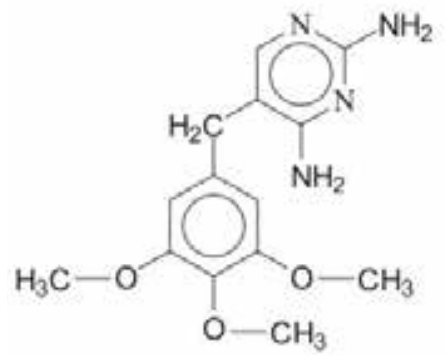

Figura 2. Estrutura molecular da trimetoprima

precisando de tempos de tratamento significativamente maiores.

Devido à imcapacidade de remoção de alguns fármacos pelo sistema de tratamento de esgoto, faz-senecessário o uso de técnicas mais sofisticadas, como os processos oxidativos avançados com o uso da irradiação com acelerador de feixes de elétrons (Silva, 2014). Para a eliminação dos resíduos farmacológicos do ambiente, há os processos de adsorção (biótico) em sólidos suspensos (lodo de esgoto) ou com carvão ativado, biodegradação (biótico) em que bactérias que agem mais no meio aquático, e fungos atuam mais no solo, degradam os compostos orgânicos, fotodegradação (abiótico) devido à exposição solar e à hidrólise (abiótico). A adsorção depende das características do fármaco como a hidrofobicidade e interações eletrostáticas, assim, o fármaco irá se agregar com partículas ou microrganismos. Fármacos com caráter ácido, como alguns AINEs (Ácido acetilsalicílico, ibuprofeno, fenoprofeno, cetoprofeno, naproxeno, diclofenaco, indometacina), o ácido clofibrico, bezafibrato e gemfibrozila ocorrem como íons em $\mathrm{pH}$ neutro) e têm pouca tendência de adsorção no lodo. Uma diminuição no $\mathrm{pH}$ aumenta razoavelmente a adsorção. Em $\mathrm{pH}$ neutro, esses compostos farmacêuticos possuem carga negativa, por isso, ocorrem principalmente na fase dissolvida no efluente (Buser et al., 1998 apoud.Tambosi, 2008). Carvões ativados podem eliminar vários compostos farmacêuticos poluentes, uma vez que a existência de anéis benzênicos ou grupos amino na estrutura da maioria desses compostos possibilita serem adsorvidos por carvões ativados (Mestre et al., 2007). Estes processos de tratamento podem eliminar completamente tais poluentes por meio da mineralização ou convertê-los para produtos que são menos prejudiciais à saúde humana e ao ambiente aquático (Tambosi, 2008).

Na via urbana, Gil \& Mathias (2005) dizem que os resíduos associados a medicamentos de uso humano podem decorrer principalmente: excreção urinária ou fecal, com consequente contaminação de esgotos por fármacos e/ou seus metabólitos; ou do descarte de medicamentos vencidos em lixos domésticos e na zona rural está associado a medicamentos de uso veterinário, os quais podem ser utilizados para fins diversos. Como exemplo, destaca-se o uso de antibióticos para promoção do crescimento, a adição de hormônios em rações e agentes antiparasitários.

Os fármacos apresentam capacidade de estabeleceram relações fortes com o solo, deixando-o por muito tempo no ambiente podendo acumular ao longo da cadeia alimentar, provocando um desequilíbrio na fauna e flora, os resíduos formados pelos fármacos depositados no meio ambiente principalmente por efluentes urbanos e industriais no início não são detectáveis (Daughton e Temes, 1999). Pequenas concentrações de alguns compostos farmacêuticos podem ser responsáveis por diversas alterações sendo, portanto, um perigo para a saúde pública (Silva 2012). Portanto, são considerados potenciais contaminantes ambientais pois, sendo compostos biologicamente ativos, são capazes de induzir os mais diversos efeitos sobre os organismos (Jorgensen et al 2000). Deve-se considerar o fato de que esses produtos fazem uma seleção dos microrganismos, deixando vivos apenas os mais resistentes (Balbino, 2011). Assim, as bactérias poderão sofrer mutações no seu material genético, tornando-as mais resistentes a várias classes de medicamentos.

Os fármacos, mesmo quando projetados para atuarem em seres humanos, afetam organismos aquáticos devido à semelhança nos receptores para determinados medicamentos, afetando a hierarquia biológica (célula, órgão, organismo, população e ecossistema) (Suchara, 2007). A presença de alguns grupos de fármacos no meio ambiente merece atenção especial, dentre eles estão os antibióticos e os estrogênios. Os primeiros, devido ao desenvolvimento de bactérias resistentes (Boletim Informativo, 2011). O aumento populacional de bactérias devido ao aumento de nutrientes disponíveis a elas, gera uma competição pelo oxigênio entre os seres aquáticos causando um desequilíbrio ecológico (Miranda, 2013). Os estrogênios, pela potencialidade de afetar adversamente o sistema reprodutivo de organismos aquáticos, causando efeito de "feminização" nos peixes machos, 
podem afetar negativamente o sistema endócrino de diversos organismos aquáticos são solúveis em gordura, assim, altos níveis podem estar presentes em carne, peixe, ovos e derivados do leite. $\mathrm{O}$ aumento crescente das concentrações dos metabólitos destes compostos, como o $17 \alpha$ etinilestradiol, mestranol e o diestilbestrol, tem sido causado pela descarga de uma grande quantidade de medicamentos expirados (não utilizados) advindos de domicílios, resíduo de hospitais e indústrias farmacêuticas (Torres 2012). Os estrogênios em seres humanos podem causar câncer em testículo, na tireoide, na mama em mulheres jovens, diminuição do número de esperma em homens férteis, e isso tem chamado a atenção da Organização Mundial de Saúde-OMS, embora não seja possível ainda identificar como os contaminantes emergentes afetam o equilíbrio do sistema hormonal dos seres vivos, o crescimento também não pode ser explicado apenas pela genética.

Os resíduos da fluoxetina, por exemplo, causam efeitos nos sistemas reprodutivo dos animais, peixes, repteis e aves, reduz a produção de esperma no homem. A análise das águas brutas, realizada na mesma pesquisa, também resultou em dados preocupantes. No Rio Atibaia, por exemplo, as amostras revelam concentrações significativas do fármaco diclofenaco e dos hormônios estradiol, etinilestradiol e progesterona (Ghiselli, 2010).

Apesar da classificação dos resíduos químicos, dos estudos realizados acerca das substâncias geradas por eles e seus efeitos sobre o meio ambiente, existe um descaso e despreparo na questão do manejo de tais substâncias em muitos lugares do mundo. Nos países desenvolvidos, existe gerenciamento de resíduos. $\mathrm{Na}$ Inglaterra e Alemanha, usa-se a incineração do lixo para produzir energia, nos EUA, o lixo é destinado a aterros sanitários adequados, incineração, reciclagem e compostagem.

Mas especificamente com fármacos, o que fazer após expirar o prazo de validade. Na maioria das vezes, por falta de orientação e alternativa, o usuário tem descartado forma inadequada $o$ medicamento no meio ambiente, ocorrendo geralmente nos vasos sanitários e no lixo doméstico.

O regime diferenciado de contratações, RDC, $n^{\circ} 33$ (Brasil, 2003) preconiza que o gerenciamento dos resíduos sólidos em saúde constitui-se um conjunto de procedimentos de gestão, planejados e implementados a partir de bases científicas e técnicas, normativas e legais. Assim, deve ser aplicado para reduzir a produção de resíduos e proporcionar aos resíduos gerados, um encaminhamento seguro, de forma eficiente, visando à proteção dos trabalhadores, à preservação da saúde pública, dos recursos naturais e do meio ambiente. Duas normas em exercício no país também tratam da questão de descarte dos resíduos gerados na área da saúde. São elas a RDC no. 306 da ANVISA (Brasil, 2004), que dispõe sobre o gerenciamento dos resíduos de saúde, e a resolução no. 358 do Conselho Nacional do Meio Ambiente (CONAMA) (Brasil, 2005), que dispõe sobre o tratamento e disposição final dos resíduos de saúde por eles gerados.

A posição desenfreada do Brasil em relação à produção de lixo requer uma atenção especial. Programas de educação ambiental são essenciais na contenção e no descarte correto do medicamento. Todas as instituições, família, escola, igreja, empregatícia, podem abraçar essa causa, assumindo responsabilidades socioeducativa em sua comunidade. Em relação à legislação, a criação de pontos para coleta dos remédios é uma forma de assegurar o gerenciamento desses resíduos, para serem encaminhados ao descarte adequado, passando, assim, a ser responsabilidade das farmácias e drogarias a destinação final desses medicamentos. As farmácias e drogarias poderão reutilizar esses medicamentos ou destiná-los a aterros sanitários próprios para a deposição e destruição de resíduos sólidos.Porém, somente com a elaboração de uma legislação eficiente e coercitiva, as presentes alternativas concretas para esse problema que o descarte correto de medicamentos poderão ser uma realidade.

\section{Legislação}

A Constituição Federal, em seu artigo 196, dispõe que "A saúde é direito de todos e dever do Estado, garantido mediante políticas sociais e econômicas que visem à redução do risco de doença e de outros agravos e ao acesso universal e igualitário às ações e serviços para sua promoção, proteção e recuperação".

Após nossa Carta Magna, o assunto foi abordado pela Lei n..$^{\circ}$ 8.080/90, que dispôs sobre as condições para a promoção, proteção e recuperação da saúde, a organização e o funcionamento dos serviços correspondentes, determinando em seu artigo 13, inciso III a necessidade de articulação de políticas públicas que tratem das atividades farmacêuticas.

Em seguida, surgiram diversas legislações no intuito de regulamentar o gerenciamento final de medicamentos. A 
associação Brasileira de Normas e Técnicas (ABNT), por meio da NBR 12.808, em 1993, classificou os Resíduos de Serviços de Saúde (RSS) em classe A (resíduo infectante - agulha e gaze); classe B (resíduo especial - químico, farmacêutico e radioativo); classe $\mathrm{C}$ (resíduo comum - papel e restos de comida), mas não se tratou sobre a questão do descarte.

Quase uma década após, a Lei n 9.966/2000 Instituiu a Taxa de Serviços Administrativos - TSA, em favor da Superintendência da Zona Franca de Manaus - Suframa, estabeleceu preços a serem cobrados pelo Instituto Brasileiro do Meio Ambiente e dos Recursos Naturais Renováveis - Ibama, criou a Taxa de Fiscalização Ambiental - TFA. A Lei n 9.974/2000 dispôs sobre a pesquisa, a experimentação, a produção, a embalagem e rotulagem, o transporte, o armazenamento, a comercialização, a propaganda comercial, a utilização, a importação, a exportação, o destino final dos resíduos e embalagens, o registro, a classificação, o controle, a inspeção e a fiscalização de agrotóxicos, seus componentes e afins. Houve ainda normas estabelecidas pelos órgãos do Sistema Nacional do Meio Ambiente (Sisnama); do Sistema Nacional de Vigilância Sanitária (SNVS); do Sistema Unificado de Atenção à Sanidade Agropecuária (Suasa); do Sistema Nacional de Metrologia, Normalização e Qualidade Industrial (Sinmetro).

Em 7 de dezembro de 2004, surge a Resolução da Diretoria Colegiada da Agência Nacional do Meio Ambiente - RDC n. ${ }^{\circ}$ 306, que dispõe sobre o gerenciamento de resíduos de serviços de saúde, classificando os em: grupo A - resíduos com risco biológico; grupo $\mathrm{B}$ - resíduos com risco químico; grupo $\mathrm{C}$ - rejeitos radioativos; grupo D - resíduos comuns e grupo $\mathrm{E}$ resíduos perfuro cortantes. Em seguida, vem a Resolução n. $^{\circ}$ 358/2005 do Conselho Nacional do Meio Ambiente-CONAMA que dispõe sobre o tratamento e a disposição final dos resíduos dos serviços de saúde e dá outras providências. Aplica-se ainda à questão dos resíduos sólidos a Lei n. $^{\circ}$ 11.445/2007 que estabelece diretrizes nacionais para o saneamento básico.

Apesar da colaboração de todos os dispositivos citados, a legislação mais abrangente estava por vir. No ano de 2010, foi promulgada a Lei n. ${ }^{\circ}$ 12.305/10, que instituiu a Política Nacional de Resíduos Sólidos, dispondo sobre seus princípios, objetivos e instrumentos, bem como sobre as diretrizes relativas à gestão integrada e ao gerenciamento de resíduos sólidos.
Como se pode observar, existe legislação acerca do tema, o problema está fato desta atribuir obrigações apenas aos responsáveis pela fabricação e distribuição à destinação final correta dos produtos existentes dentro do seu estabelecimento. Estes, por sua vez, não possuem obrigação legal de recolher os fármacos que sobram dos produtos que vendem, gerando um enorme problema, o descarte incorreto de medicamentos pela população.

\section{Conclusão}

Existe hoje a necessidade do desenvolvimento e utilização de novos processos de tratamento de efluentes, devido à ineficiência dos tratamentos convencionais, que garantam uma boa remoção empregando técnicas capazes de identificar e quantificar estes compostos, bem como buscar e utilizar processos capazes de degradar esse tipo de poluente. Parte dos resíduos farmacológicos encontrados no meio ambiente, principalmente, no meio aquático, são lançados pelos esgotos residenciais. Portanto, há a necessidade de conscientizar a população com implantação de projetos municipais em relação à forma adequada de descarte de medicamento, ao uso racional desses e ao não abandono dos tratamentos ou a auta medicação, definição de locais para a coleta e, consequentemente, o descarte adequado. Estas são as principais causas dos descartes residenciais.

\section{Referências Bibliográficas}

1. Lei n. ${ }^{\circ} 8.080$ de 19 de setembro de 1990. Dispõe sobre as condições para a promoção, proteção e recuperação da saúde, a organização e o funcionamento dos serviços correspondentes e dá outras providências. Planalto. 2015.

2. Resolução n. ${ }^{\circ} 358$, de 29 de abril de 2005. Dispõe sobre o tratamento e a disposição final dos resíduos dos serviços de saúde e dá outras providências. Ministério do Meio Ambiente. 2015.

3. Lei n. ${ }^{\circ} 12.305$, de 2 de Agosto de 2010. Institui a Política Nacional de Resíduos Sólidos; altera a Lei no 9.605, de 12 de fevereiro de 1998; e dá outras providências. Brasília: DOU, 3 ago. 2010 .2015.

4. Américo, J. H. P.; Isique, W. D.; Minillo. A.; Carvalho, S. L.: TORRES, N. H. Fármacos em uma estação de tratamento de esgoto na região Centrooeste do Brasil e os riscos aos recursos hídricos. Revista Brasileira de RecursosHídricos, Porto Alegre, v.17, n.3, p.61-67, 2012

5. Andreozzi, R.; Rafaelle M. Pharmaceuticals in STP effluents and their solar photodegradation in aquatic environment. Chemosphere. v.50, n.10,p.1319-1330,2003. 
6. ANVISA. Resolução da Diretoria Colegiada $-\mathrm{RDC} \mathrm{n}^{\circ}$ 306, de 07 de dezembro de 2004. Dispõe sobre o Regulamento Técnico para o gerenciamento de resíduos de serviços de saúde. DOU, 10 dez. 2004

7. ANVISA. Medicamentos: Conceitos Técnicos. 2015

8. Baibino, Michele Lucas Cardoso; Balbino Estefane Cardoso. O descarte de medicamentos no Brasil: Um olhar socioeconômico e ambiental do lixo farmacêutico, SP,2010.

9. Balbino, Estefane Cardoso; Balbino, Michele Lucas Cardoso. O descarte de medicamentos no Brasil: Um olhar socioeconômico e ambiental do lixo farmacêutico. In: Âmbito Jurídico, Rio Grande, XIV, n. 86, mar 2011.

10. Buser H.r., Poiger T., Müller M.d. Occurrence and fate of the pharmaceutical drug diclofenac in surface waters: rapid photodegradation in a lake. Environ. Sci. Technol., v.32, p.3449-3456, 1998.

11. Boletim Informativo Do Cim-Rs. Prática profissional: Descarte de medicamentos Maio 2011. Disponívelem: www.ufrgs.br/boletimcimrs

12. Bound J.p.; Voulvoulis N. Pharmaceuticals in the aquatic environment-a comparison of risk assessment strategies. Chemosphere, v.56,p.1143-1155,2004.

13. Brasil. Constituição (1988). Constituição da República Federativa do Brasil. In: Vade Mecum: Profissional e Acadêmico. Organização dos textos Antonio Luiz Toledo Pinto, Márcia Cristina Vaz dos Santos Windt e Lívia Céspedes. 4. ed. São Paulo: Saraiva. 2007.

14. Conama. Resolução no 316 , de 29 de outubro de 2002. Dispõe sobre procedimentos e critérios para o funcionamento de sistemas de tratamento térmico de resíduos. DOU 20 nov. 2002.

15. Doughton, C.g.; Ternes,T.a. pharmaceutical and Personal Care Products in the Environment: Agents of Subtle Change? Environmental Health Perspectives 107. 1999.

16. Ferreira, J.a. Resíduos Sólidos e Lixo Hospitalar: uma discussão ética. Caderno de Saúde Pública, v.11, n.2, 1995.

17. Gil, E. De S.; Mathias, R.o. Classificação e riscos associados aos resíduos químicos Farmacêuticos. Revista Eletrônica de Farmácia, v.02, 2005.

18. Glassmmeyer St, Hinchey Ek, Boehme Se, et al. Disposal practices for unwanted residential medications in the United States. Environ Internat. 566-72. 2009.

19. Hoppe Taise Raquel Grings, Araujo Luiz Ernani Bonesso. Contaminação do Meio Ambiente pelo Descarte Inadequado de Medicamentos Vencidos ou não Utilizados. v 6, P.1248-1262. 2012.

20. Ikehata K., Naghashkar N.j., El-Din M.g. Degradation of aqueous pharmaceuticals by ozonation and advanced oxidation processes: a review. Ozone: Sci. Technol., v.28, p.353-414, 2006.

21. Jorgensen, S. E.; Halling-Sorenses, B. Drugs in the environment, Chemosphere, 40 (7) p. 691-699. 2000.

22. Lages, Andreína da Silva. Presença ambiental de resíduos fármacos: fontes, concentrações, efeitos potenciais e formas de tratamento. Faculdade Fernando Pessoa Porto, 2011.

23. Melo, Silene Alessandra Santos; Trovó, Alam Gustavo; Bautitz, Ivonete Rossi; Fernandes, Raquel. Degradação de fármacos residuais por processos oxidativos avançados. Departamento de Química Analítica, Instituto de Química de Araraquara, SP. 2008.

24. Merck, Cloridrato de fluoxetina. 2015

25. Miranda, Amanda Carvalho. Desenvolvimento de um método para monitoramento da contaminação do solo pelo fármaco azitromicina uma abordagem sustentável. Dissertação mestrado-UNINOVE, São Paulo, 2013

26. Mestre A.s., Pires J., Nogueira J.m.f., Carvalho A.p. Activated carbons for the adsorption of ibuprofen. Carbon, v.45, p.1979-1988, 2007.

27. Napoleao, D. C; Silva, R.f.; Silva, P.t.s.; Benachour, M; SILVA, V.L.: Degradação de contaminante emergente (ácidoacetilsalicílico) utilizando processo foto-fenton. ABES - Associação Brasileira de Engenharia Sanitária e Ambiental. Pe.

28. Reis Fliho, R.w; Barreiro J.C. Fármacos ETEs e Corpos Hídricos. RevistaAmbi-Água, Taubaté, v.2, p.54-61,2007.

29. Silva, Pedro Filipe Ramos. Resíduos de medicamento nos RSU: Riscos e consequências. Universidade Fernando Pessoa. Porto 2012

30. Silva, Vanessa Honda Ogihara. Avaliação da toxicidade e da degradação do fármaco cloridrato de fluoxetina, em solução aquosa e em mistura com esgoto doméstico, empregando irradiação com feixe de elétrons. Instituto de pesquisas energéticas e nucleáres, SP. 2014.

31. Sodré, F. F.; Pescara, I. C.; Montagner, C. C.; Jardim, W. F. Assessing selected estrogens and xenoestrogens in Brazilian surface waters by liquid chromatography-tandem mass spectrometry. MicrochemicalJournal, New York,v. 96, p. 92-98, 2010.

32. Suchara, E.A. Desenvolvimento de Metodologias Analíticas para determinação de fármacos em fluídos biológicos e amostras ambientais por cromatografia líquida e gasosa. Florianópolis, 2007.

33. Tambosi, José Luiz. Remoção de fármacos e avaliação de seus produtos de degradação através de tecnologias avançadas de tratamento. Universidade Federal de Santa Catarina. Florianópolis, 2008.

34. Torres, N.h.; Romanholo Ferreira, L.f.; Americo, J. H. P.; Freguglia, R. M. O.; Moura-Andrade, G. C. R.; Tornisielo, V. L. Analysis and occurrence of residues of the hormones estriol, 17alpha-ethinylestradiol and 17 beta-estradiol in urban water supply by HPLCDAD. IOSRJEN JournalofEngineering, v. 2, p. 984-989, 2012.

35. Vieira, Karla Moreira, Aplicação dos processos oxidativos, redutivos e (foto) eletroquímicos na degradação de fármacos em meio aquoso. MG, 2011.

\section{Euzani S. Duarte; Gislene C. S. de Aquino* \& Rogério G. Lima}

Centro Universitário De Anápolis - UniEVANGÉLICA - Av. Universitária Km. 3, 5 - Cidade Universitária, Anápolis - GO, 75083-515

*E-mail: gislene_sousa2@yahoo.com.br 